\title{
Wavelets Application in Prediction of Tunnel Defects in Friction Stir Welding of Alloy Joints from Vibroacoustic ANN-Based Model
}

\author{
S.Lakshman kumar, Saranya S.N., Raj Jawahar.R
}

\begin{abstract}
This paper examines a connection of acoustic emission signal to the core parameters of the friction stir welding process, based on the artificial neural networks (ANNs). AE Instrument NI USB-9234 has obtained acoustic $Z$ and $Y$ emission signals. Wavelet Transform was used as the ANN's output through numerical and time parameters for discomposed acoustic pollution signals. The ANN inputs include rotation speed and frequency of the device, the machine profile and the tensile strength parameters. A multi-layer neural feed-forward network was selected and trained using the Levenberg Marquardt algorithm for different network architectures. Ultimately, an overview will be provided of the correlation between the estimated and analyzed results. The prototype obtained can be implemented with the aid of acoustical emission signals to design and improve automated system parameters and mechanical properties of the joint.
\end{abstract}

Keywords: Wavelet Application, Prediction, Tunnel, NI-USB-9234, ANN-Based Model.

\section{INTRODUCTION}

Friction stir welding (FSW) is an energy intensive, environmentally friendly and flexible joining process, developed in 1999 by the Welding Institute of the UK and has proved to be one of the most important achievements in the field of aluminum alloys joining. [1].

The main advantage is that products that are hard to weld or not combine through a fusion soldering system can be effectively joined [2]. For storage, boats, seafaring, fuel tanks and the food-saving field FSW were commonly utilized. [3].

In the ways that allow us escape the complexities of the research phenomena, science and technology design have helped to develop models of similar behaviors to the physical process. On this basis, and because friction stir welding is widely recognized as a reliable soldering technique, different online testing methods for modeling and quality control are expected to be further established. [4].

ANEM is a non-destructive measurement tool for real time applications. Acoustic Emission Monitoring (AE) [5] it is characterized by a detailed description of the actions of anomalies, but its large nature should be taken into account. The use of non-destructive technology (NDT) in mechanical

Revised Manuscript Received on November 12, 2019

* Correspondence Author

S.Lakshman kumar, Assistant Professor, Department of Mechanical Engineering, SONA College of Technology, Salem, India

Saranya S.N., Research scholar, Department of Process Dynamics and Control, Coimbatore Institute of technology, Coimbatore, India

Raj Jawahar.R, Researcher, Department of Mechanical Engineering, Dynamechz Design Solutions, Chennai, India. and manufacturing processes is illustrated through different reports.

Roca et al. and Macias et al. demonstrated the advantages of using $\mathrm{AE}$ as no interruption to characterize the reliability of the arc gas welding system. A new index for the GMAW stability study of arc-generated AE processes was created. In this work. The investigators have checked the usefulness of the proposed method as a new way of assessing soldering stability.

The effectiveness of applying AE to power FSW systems was shown by Fernandez et al. In aluminium AA 1050, the researchers developed a system to determine the impact of the device profile on the resistance stir solding cycle using acoustic emission signals.

Soundararajan et al. have defined the correlation of the instrument's contact losses with a workpiece using the AE signal characteristics of the Fast Fourier Transform (FFT), the STFT and DWT. The researchers investigated the use of FSW system control AE approaches. We also demonstrate that $\mathrm{AE}$ is sensitive to changes in the scope of output of the device in the frequency domain.

In the study of the FSW cycle, Suresha et al. verified the applicability of vibroacousticsignals. These researchers created a theoretical template for correlating the parameters of the FSW method, device layout, product flow pattern, mechanical properties and microstructures.

The systems of welding are mechanically fluid, nonlinear and flexible in nature and have a great number of input parameters; the behavior of these processes does not have an enclosed mathematical model. In recent years the method of modeling numerous complex processes has also been employed, on the other, artificial intelligence techniques and especially neural networks (ANNs).

In a number of applications, wavelets can be used to design systems, monitor and control ANNs. These can also be used to calculate parameters which cannot usually be calculated online. In genetics, telecommunications, informatics, mathematics and engineering, $\mathrm{ANN}$ is one of the most important fields of research.

ANN can learn and generalize complex (interpolating) interactions between the variables of input and output of such programs. Recently, computer-aided ANN simulation has become increasingly important in the field of engineering. The literature shows different ANN advances related to systems

In order to estimate the mechanical properties of welding, the authors of the Okuyucu et al. used the FSW ANN-based aluminum model to use systems parameter as design output. 


\section{Wavelets Application in Prediction of Tunnel Defects in Friction Stir Welding of Alloy Joints from Vibroacoustic ANN-Based Model}

To predict FSW aluminum alloy tensile strength AA7039, Lakshminarayanan and Balasubramancontrasted the response-surface technique with the neural artificial network models. The researchers employed system variables such as rotational speed, welding speed and axial resistance as design output.

Tansel et al. conducted the study with ANN to determine the optimal operational status of the FSW program. In an excellent method for FSW optimization, the investigators used five separate ANNs to establish a correlation among two equivalent input parameters and the properties of the welding field.

The comparison of mechanical characteristics of the joint was a focus of all models based on ANN previous for FSW (tensile power, stiffness, etc.) obtained by the system parameters (tool rotational speed and movement rate) of mechanical tests and microstructural characteristics.

In existing literature, a template cannot be found that forecasts system value parameters and the mechanical characteristics of non-invasive techniques such as EA. ANS-based models previously obtained for FSW were all based upon comparisons. The new framework shown in this paper aims to address this weakness by analyzing in depth the approaches and results extracted from the analysis and development of the systemic ANN characteristics of joint vibration signals. These findings often indicate the opportunity to develop a model based on acoustic emissions to monitor and control adjustments in the parameters of FSW processes and to predict the joint mechanical characteristics.

\section{EXPERIMENTAL WORK}

The AA1050 H24 sheet is used in this journal in its dimensions of $200 \mathrm{~mm}(\mathrm{~L}), 100 \mathrm{~mm}(\mathrm{~W})$ and $3 \mathrm{~mm}(\mathrm{~T})$. It is recorded and commonly used in automobile cutting systems, chemical and food processing equipment, light reflector and heat exchanger modules. The electrical behavior of this alloy is understood. In the AA1050 H24 the chemical compositions were of $0.25 \mathrm{Si}, 0.40 \mathrm{~F}, 0.05 \mathrm{Cu}, 0.05 \mathrm{Mn}, 0,05$ $\mathrm{Mg}, 0.07 \mathrm{Ti}, 0.03,99.05 \mathrm{Al}$. The chemical compositions used for testing (wt percent $\mathrm{t}$ ). For the FSW operation a CNC instrument with a displacement control was used. The plates are linked with the ass by FSW, and locked with mechanical clamps in place. The angle of travel used was 0 tons and the welding path became normal.

Various test runs are carried out in order to determine practical working limits of FSW system parameters. The acquisition of faultless joints within the experimental area has been tested in order to carry out this analysis. The life of the instrument was also regarded. During the measurements it was assured that the device retained its proportions.During the test, there were three speed levels $(40,63$ and $100 \mathrm{~mm} \cdot \mathrm{min}-1)$ and the rotation speed of the tools $(450,710$ and $1120 \mathrm{rpm})$. Experimental factory-type models were also used, including 18 welding tests. Both weld tests take place in the same conditions three times in order to reduce test failure.

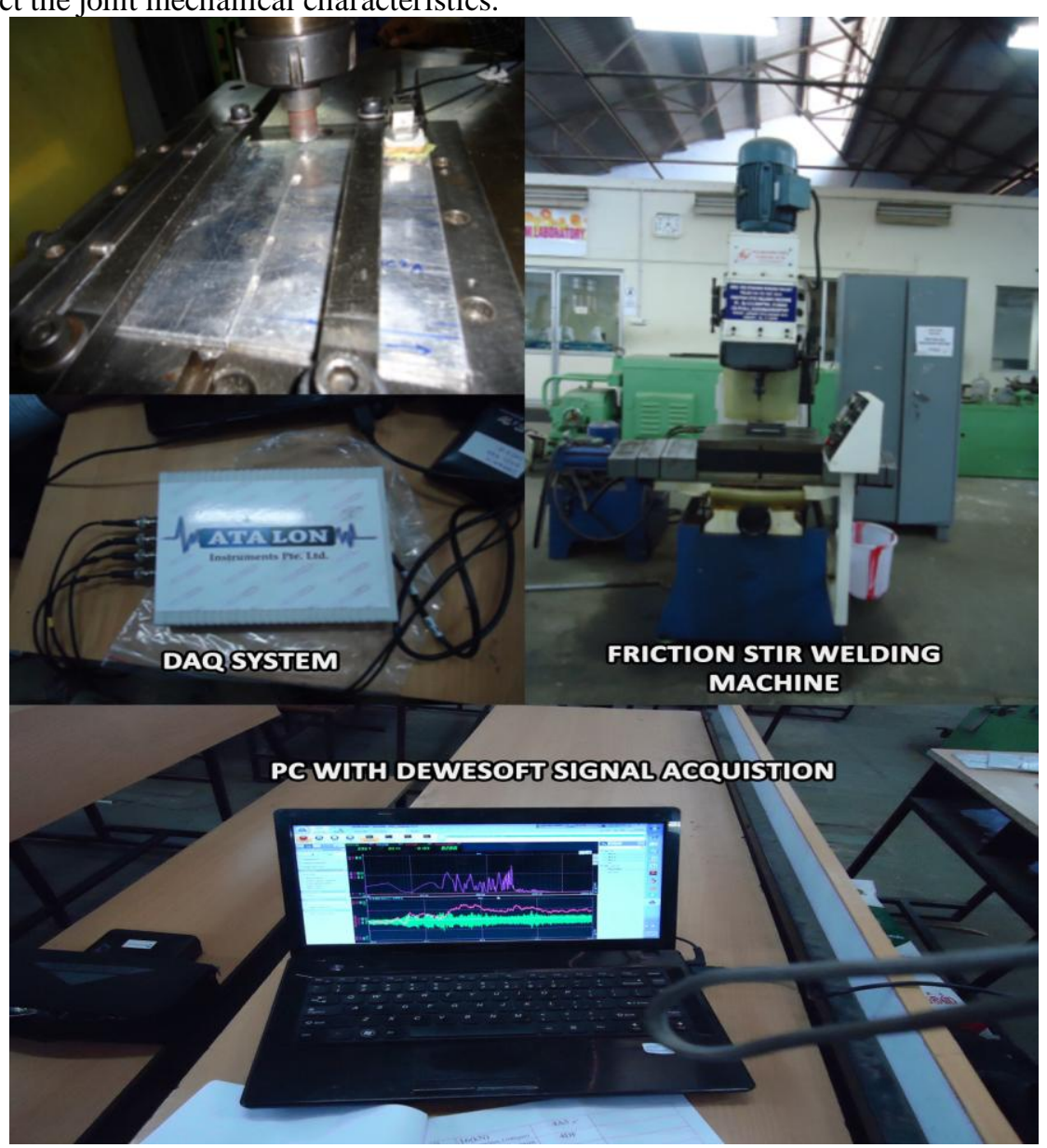

Fig.1 Experimental photograph of the condition monitoring in FSW process 
The machine specimens and shoulder diameters are 3 and 10 $\mathrm{mm}$. A traditional cylinder pin with grooves (Tool1) and a threaded cylinder pin (Tool2) are taken as two separate pin profiles. Both devices have a concave shoulder profile. During the soldering process, the vibroacoustic signals produced in $\mathrm{Z}$ and $\mathrm{Y}$ directions were collected and recorded with the USB-9234 connected AE instrument. Until welding, the AE signals from the external noise are tracked. All signals were analyzed using MATLAB technology and were sampled at $51.2 \mathrm{kHz}$. The rear panel was mounted perpendicularly on the top and side surfaces of two acoustic emission sensors (piezoelectric based). The sensors were placed on the back plate and connections were used so that the back plate was well acoustic connected to the detector.

Table: 1 Welding parameters and dimensions

\begin{tabular}{|c|c|}
\hline Parameters & Value \\
\hline Tool rotation speed & $1100 \mathrm{rpm}$ \\
\hline Axial force & $11 \mathrm{KN}$ \\
\hline Feed rate & $100 \mathrm{~mm} / \mathrm{sec}$ \\
\hline Plunge depth & Cylindrical pin \\
\hline Tool type & High speed steel grade HSS M2 \\
\hline Tool Material & mm \\
\hline Work piece & Al6063 aluminum alloy Size: $150 \times 75 \times 6$ \\
& Defect free \\
\hline Experiment 1 & Pre Induced Tunnel defect \\
\hline Experiment 2 &
\end{tabular}

Vibroacoustic sound capture characteristics. AE signal processing has a major task in selecting a suitable frequency range to provide data on device actions. To better describe the data obtained, an external statistical analyzes are necessary.

In this article, the numerical parameters and the Wavelet Transform (WT) of the vibroacoustic signal are used to obtain information from the ANN used. The results are collected. The mean value, normal variance and coefficient of variance are the most significant parameters in welding analysis.

WT is a powerful tool for decomposing non-stationary signals like vibroacoustic signals. WT permits long periods in which reliable low-frequency data is required and shorter places in which information of high frequency is necessary. In this research, eight waves of Daubechies were used to split signal for different scales to hit the same bands that are not appropriate for testing.

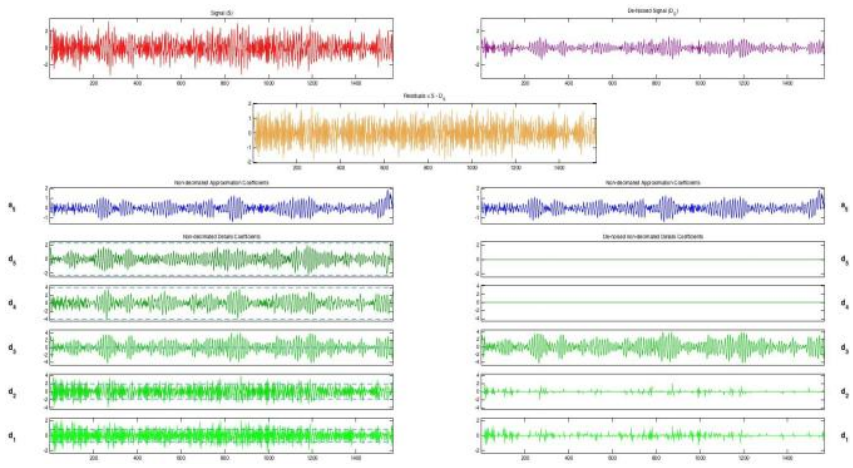

Fig.2 Signal denoising and filtration using un-decimated wavelet transform
In this situation, the operation of WT is equivalent to a filter of frequency bands of frequency[ $0,100 \mathrm{~Hz}],[100,200 \mathrm{~Hz}]$, [ 200, $400 \mathrm{~Hz}],[400,800 \mathrm{~Hz}],[800 \mathrm{~Hz}, 1.6 \mathrm{kHz}],[1.6,3.2$ $\mathrm{kHz}],[3.2,6.4 \mathrm{kHz}],[6.4,12.8 \mathrm{kHz}]$ and[ $12.8,25.6 \mathrm{kHz}]$. The order (eight) of the WT was associated with this frequency range. Chen et al. has shown that the WT acoustic pollution measurement is accurate while evaluating 6061 FSW aluminum. The researchers demonstrated that the electrode penetrates or removes the weld joint and touches the shoulder and separates the surfaces by the major changes in the power of the unit. As a consequence of the decomposition of WT, statistically the signal produced was defined in the different energy bands. In the end, a critical experiment was carried out in order to extract the numerical parameters which revealed substantial variations in their implementation as the ANN data.

Table 2. Parameters of artificial neural network process for RMS

\begin{tabular}{ll}
\hline Name & ANN model forRMS \\
\hline Networktype & FFBP model \\
No ofhiddenlayers & 2 \\
& \\
Transferfunction & PURELIN \\
Trainingfunction & TRADNLM \\
Learningfunction & LEARNGDM \\
Performancefunction & Mean square error \\
Numberofneurons & 4 \\
Sum ofsquared error & 0.00073676 \\
Numberofepochs & 6 \\
Validationchecks & 2 \\
Learningfactor & 0.6 \\
\hline
\end{tabular}

Artificial Neural Network Methodology. The following approach was extended for observations on the efficacy of 


\section{Wavelets Application in Prediction of Tunnel Defects in Friction Stir Welding of Alloy Joints from Vibroacoustic ANN-Based Model}

ANN for assessing improvements to the system and mechanical characteristics by the parameters of FSW and machine-generated vibroacoustic signals. In this approach, a neural network has been developed that detects and explains the communication between the vibroacoustic stimuli. The multilayered perception (MLP) neural network was one of the most common and early established engineering network architecture. The number of layers and nodes in individual layers determines the neural network architecture. A back-up (BP) type, normally a two-stage generic BP network was selected in this study. Equation (1) is the ANN output capability used in which W1 and W2 are used to describe the secret intake matriximum and output neurons visible, b1 and b2 are the input vectors of each of the hidden layer and output layer neuron. The framework has three rates that are respectively source, stored and output sheets.A variety of output nodes named neurons is involved in the input layer and all input variables. The characteristics of the vibroacoustic stimuli correlate in this situation. The values of $\mathrm{x} 1$ to $\mathrm{xn}$ have been defined. In one invisible step, data from the output layer is stored. The weighted reference (W1) in every neuron in the secret layer is linked to each of the previous layer's neurons. Such weighted average of a non-linear activation function is distributed to generate the vector $(y)$ output of $0-1$. The MLP is particularly suitable to model non-linear connections between the produced vibroacoustic signals and the FSW parameters. Transfer functions in terms of sigmoid mechanism of movements of all secret nerves.

$$
f(x)=\frac{\exp (x)-\exp (-x)}{\exp (x)+\exp (-x)} \cdots
$$

In the output layer the FSW parameters include a neuron for each variable to be modeled is determined. Again, in the previous secret layer of the neuron (W2), weights are used to link each neuron production. The neurons in the unseen layers are computer units which not linearly track input and output. The ANN (y) output value is compatible with the welding parameters defined. The transfer functions are linear for the output neurons as shown in Formula 2.

$$
f y=x \ldots \ldots \ldots \ldots
$$

In the development of neural networks it is important to determine the amount of secret neurons. An excess of occult neurons usually gives too much flexibility to overfit. Too few unknown neurons on the other side limit the ability of a network to learn and weaken its approximation.

From examples, that is the patterns, ANN learns. It had to be structured with a preprocessing stage in order to incorporate trends into the ANN. To develop and evaluate a neural network, information and the corresponding goal values are needed. 126 patterns in learning quality and 18 were used in a sample set.

Neural neural network architectures for learning. The neural network was trained using two different trainingalgorithms:
(1) Gradient descent and dynamic algorithm, Levenberg-Marquardt algorithm.

BP programming uses a gradient descent algorithm that eliminates the average error between goal data and projections for the nerve network. One of the biggest problems with simple BP (gradient descent algorithm) was the extensive training time required. The Levenberg-Marquardt BP algorithm (LMBP) comprises two main categories, heuristic methodologies and standard numerical optimization techniques.

The LMBP algorithm is equivalent to the nearly modern one by which the Hessian matrix (second derivatives) is increasing. When the value formula is like a sum of quadratic a, the Hessian matrix $\mathrm{H}$ can be determined

$$
H=
$$

And the gradient $\mathrm{g}$ can be determined as

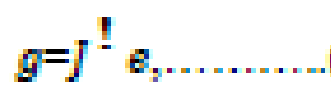

In $\mathrm{J}$ the Jacobian matrix representing the first derivatives of network errors in weight and biais and e is a network error function. The Jacobian matrix can be measured using a generic back-propagation procedure that is much less difficult than the Hessian matrix. In the case of moderate-sized networks, the LMBP algorithm was tested. Equation (6) demonstrates the role used during ANN learning:

$$
F(w)=\frac{1}{2} e^{T}(w) \cdot e(w) \ldots \ldots \ldots(5)
$$

By accordance with the successes of these two learning algorithms, it is decided the algorithm is greater than the other. The test data were inserted into the ANN for analysis of the output achieved after analysis of functionality of different network architectures. Figure 2 demonstrates the total methodological measures used in this analysis.

Before being given to the neural network, the preprocessing phase creates the conversion of inputs and goal information. On the other side, after storage the optimized items are allocated to the same units used for the initial objectives.

Based on the knowledge of the latest obtained prototype, the values of the unit parameters cannot be determined in the trials using a vibroacoustic signal. This design would allow the development of future applications, based on vibroacoustic signals produced using this method, to monitor and check FSW system parameters automatically.

\section{RESULTS AND DISCUSSION}

A non-destructive method for measurement of real time detection systems is acoustic pollution. All the vibroacoustic signals are described in this study. In order to compare these 
with device variable and structural properties of the FSW joint, the ANN simulation has been performed by obtaining the FSW vibration signal attributes.

The crossing perpendicular to the welding direction is indicated by the two representational requirements of the tool speed $=710 \mathrm{rpm}$, speed $=40 \mathrm{~mm}-1$ (Figure 4(a), condition 1) and speed $=63 \mathrm{~mm}-1$ (Figure 3(c), condition 2). The following are shown on figures: 3(a) and 3(c) Figure 2.

The intersection perpendicular to the line of solder reveals two symbolic conditions with the device speed $=710$, speed $=$ $40 \mathrm{~mm}-1$ (Figure 3(a), condition 1) and speed $=63$ mm.min-1, (Figure 3(c), condition 2). The following are shown on figures: 3(a) and 3(c) Figure 2.
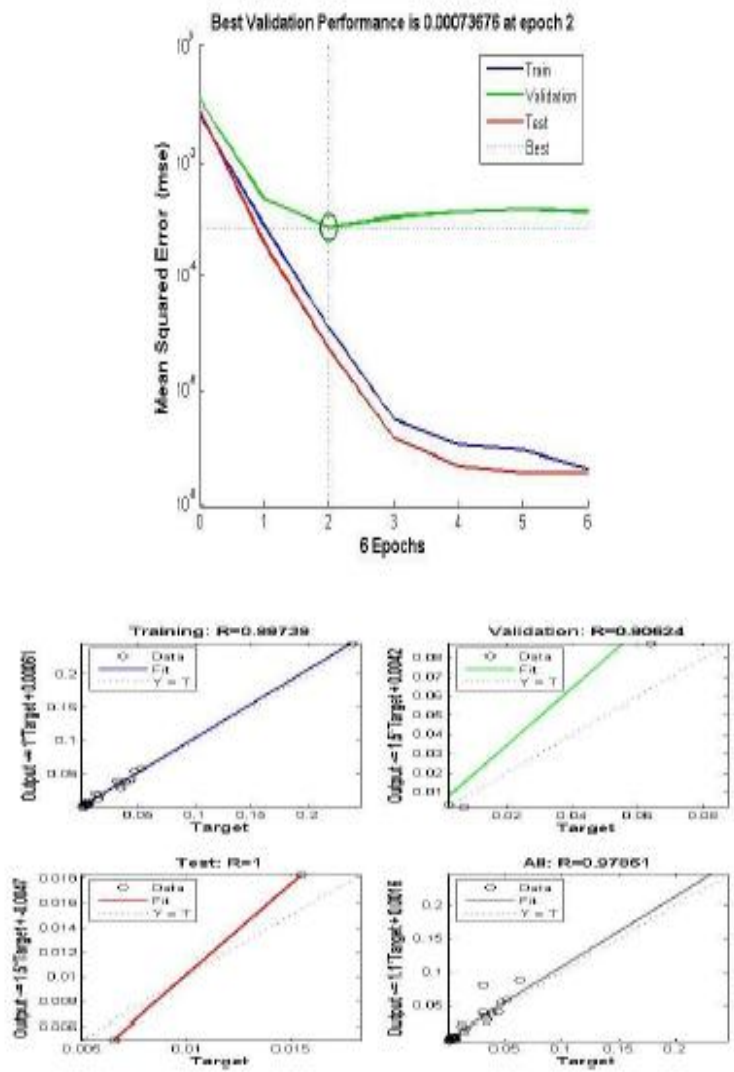

Figure 3. Performance and regression plots for RMS

Table. 3 Process parameters and their levels

\begin{tabular}{|c|c|c|c|c|}
\hline \multirow[t]{2}{*}{ SNo } & \multirow[t]{2}{*}{ parameters } & \multicolumn{3}{|l|}{ levels } \\
\hline & & -1 & 0 & 1 \\
\hline 1 & Defects & Tumel & Void & Weld flash \\
\hline 2 & Spindle speed rpm & 1000 & 1100 & 1200 \\
\hline 3 & Feedratemm/min & 50 & 75 & 100 \\
\hline
\end{tabular}

Table.4 Prediction of RMS of the identified defects in FSW process

\begin{tabular}{|c|c|c|c|c|c|}
\hline \multirow[t]{2}{*}{ Defects } & \multirow[t]{2}{*}{ Speed } & \multirow{2}{*}{$\begin{array}{l}\text { Feed } \\
\text { rate }\end{array}$} & \multicolumn{2}{|l|}{ RMS } & \multirow[t]{2}{*}{$\%$ Error } \\
\hline & & & Exp & $\begin{array}{l}\text { ANN } \\
\text { predicted }\end{array}$ & \\
\hline Void & 1100 & 75 & 1.127 & 1.201 & 5.1887 \\
\hline Void & 1200 & 75 & 1.286 & 1.281 & 1.7483 \\
\hline Weld flash & 1100 & 75 & 1.292 & 1.284 & 2.7397 \\
\hline Weld flash & 1200 & 100 & 1.382 & 1.375 & 1.8325 \\
\hline Void & 1000 & 75 & 1.261 & 1.257 & 1.5326 \\
\hline Void & 1100 & 75 & 1.262 & 1.260 & 0.7634 \\
\hline Tunnel & 1200 & 50 & 1.215 & 1.211 & 1.8605 \\
\hline Void & 1100 & 50 & 1.226 & 1.220 & 2.6549 \\
\hline Void & 1100 & 100 & 1.212 & 1.241 & -13.6792 \\
\hline Void & 1100 & 75 & 1.312 & 1.306 & 1.9231 \\
\hline Void & 1100 & 75 & 1.109 & 1.124 & -13.5031 \\
\hline Tunnel & 1000 & 50 & 1.189 & 1.181 & 4.2328 \\
\hline Void & 1100 & 75 & 1.385 & 1.369 & 4.1558 \\
\hline Tunnel & 1000 & 100 & 1.412 & 1.396 & 3.8835 \\
\hline Weld flash & 1200 & 50 & 1.391 & 1.399 & -2.0456 \\
\hline Tunnel & 1100 & 75 & 1.374 & 1.399 & -6.6841 \\
\hline Weld flash & 1000 & 50 & 1.385 & 1.399 & -3.6360 \\
\hline Void & 1100 & 75 & 1.372 & 1.399 & -7.2576 \\
\hline Tunnel & 1200 & 100 & 1.413 & 1.399 & 3.3902 \\
\hline Weld flash & 1000 & 100 & 1.395 & 1.399 & -1.0123 \\
\hline
\end{tabular}

Extraction of Vibroacoustic Signals. The reduction of noise was the first stage in the characterisation of $\mathrm{AE}$ signals and the effects in previous work, as stated in the previous section. The analysis of vibroacoustic signal characteristics was based on statistics. Various methods are used to evaluate the properties of these signals as an ANN source. In this essay the Variance Analysis (ANOVA) is a way for evaluating the statistical results of welding feature changes (ANN outputs). The vibroacoustic signals in accordance with conditions 1 and 2, can be displayed in Figure 4. Such parameters are similar to the rotation speed of the tool $=710 \mathrm{rpm}$, and have the same machine profile. The flow rate was $40 \mathrm{~mm} . \mathrm{min}-1$ (Figure 4(a)) and 63 mm.min - 1, respectively (Figure 4(b)). The vibroacoustic pulse frequency is greater in condition 1 due to a lower propagation rate $(40 \mathrm{~mm} . \mathrm{min}-1)$. As can be seen in Figure 4. The finding that ANOVA is extended to all vibroacoustic stimuli indicates that the improvements in the parameter rates affected only two functions. In this scenario, the ANN output was chosen for the information median and the RMS. The numerical parameter values for the signal analyzes shown in figure 4 are shown in Table 1.

Considering the complex nature of the vibroacoustic signals provided by the FSw device, the comparison between the vibroacoustic signal and the process parameter is not adequate to use two vibroacoustic properties alone. This research thus indicates that additional functions based on the time-frequency properties of vibroacoustic signals with a WT be employed to produce statistical functions that suit the various signal ranges.

WaveletTransformforNewFeatures. The DWT is used for obtaining specific statistical characteristics of each vibroacoustic signal sequence. The DWT comprises of a series of signals decomposed from different frequency bands. The diagrams in Figures 5(a) and 5(d) show the WT of the vibroacoustic signal state 1 and 2 . In this analysis, eight rates 


\section{Wavelets Application in Prediction of Tunnel Defects in Friction Stir Welding of Alloy Joints from Vibroacoustic ANN-Based Model}

of DWT decomposition and mother wavelet Daubechies (Db5) are used.

The vibroacoustic signal can be measured in Figure 5 for each decomposed frequency band referring to Condition 1 and 2. Visual inspection of these figures allows the observation for each similar frequency band of the differences between the signals. The F-test was used to determine vibro-acoustic properties influenced by FSW parameter shifts. The study did not take into account the bands of amplitude correlated with the distortion which were excessively shown in the previous epigraph.The findings of the ANOVA methods for three chosen properties, taken from DWT decomposition, used for each of the welding parameters as an output ANN reveals in Figure 6.

Following the statistical analysis of each decomposed signal by ANOVA, 14 new input functions of the ANN have been chosen. The established outputs display significantly different means of groups because of changes in the system parameters.

Neural Network. ANNs have been used in a variety of fields in recent years and have been able to provide approaches to science and industrial issues. Each article demonstrates the features that are highly significant in the ANN feedback of the vibroacoustic signals chosen. Also provided are the findings of the chosen topology and learning algorithm.

For increasing the amount of inputs (14 inputs) for ANN, sensitivity and main component analysis were established. This technique decreases the vector output dimension without redundant components. No more than 10 function values (x1,., x10, etc.) were selected. Six of the time domain signals were derived and eight quantitative parameters were taken from decomposed DWT signals. In this case, Table 2 shows the vibroacoustic properties selected as the ANN input. The changes in welding processes and tensile strength have a significant effect on these characteristics.

Table 3 contains three neural network options which have been architecturally tested. The study of specific computational and training algorithms illustrate the good quality and less final errors in the Levenberg-Marquardt algorithm for a network with one hidden layer (16 neurons). The chosen network configuration is composed of 10 input neurons, 16 hidden neurons with a non-linear (log sigmoid) activation mechanism and three output neurons with a linear activation feature (device rotation frequency, motion speed and system profile). The chosen network 10-16-3 topology is shown in Figure 7.

There was no consistency between the ANN with a smaller number of the layer nodes, and the pattern error was high. The final correct evaluation was obtained for architecture 10-16-3 at $99.9 \%$. With this weight formula, the correct outcomes could be obtained using $95 \%$ confidence. Use more than the appropriate number of hidden network layers may lead to an unwanted information overload.

Neural Network Evaluation. New tests of the neural network architecture were produced to affirm the generalization potential of the network. The weights and bias values are replaced in (8) that control the function of the ANN after the values have been obtained. After the learning data set has been educated, the networks are tested to insure that they can meet the FSW system parameters and learn power using the information check.

The ANN output values are applied to the post-processing phase to obtain the real value of the systems parameters. Figure 8 shows the correlation between mechanical characteristics of the FSW joint and parameters determined with an ANN. As a consequence of the regression analysis, the correlation function obtained is also shown. The observed and projected performance values are similar to each other, as can be seen in Figure 8. The analysis of the ANN with the information from the test shows that the FSW parameters correspond practically linearly to the ANN's parameters.

The comparative comparison between an ANN-based model's tensile strength and that calculated by a mathematical model that was established during previous work is shown in Figure 8(c). The meaning of the modified determinative coefficient $\mathrm{r} 2$ was 91.4 percent for the mathematical model in this situation, which suggests that the method is not understood by less than 9 percent of the total variations. In $99 \%$ of situations, the neural network design predicts traction opposition. The great advantage of using the ANN is that it is easy, due to the generalization power of the ANN, to infer further parameter values from the vibroacoustic signal. Table 4 presents the findings of ANN details on both parameters, compared to actual evidence. The results show that the ANN description for the layout of FSW device parameters is correct.

\section{CONCLUSIONS}

This paper assesses the probability of ANN using the system signals produced by the method to predict the FSW process parameters. The result was that

(i) Online vibroacoustic signals processing provides useful information on changes in the FSW process parameters and the mechanical properties of joint based upon statistical characterization of a specific frequency range;

(ii) The results show that the current ANN model is an effective way of forecasting FSW system parameters and joint tensile strength based on vibro-acoustic signal measurements;

(iii) A new method for the nondestructive simulation of welding and mechanical properties is possible to predict, without intervening in development process;

(iv) The Levenberg-Marquardt algorithm has higher performance than declining momentum in this case;

(v) A vibroacoustic signal analysis showed the application of the ANN to model dynamic connections, parameters and responses;

(vi) RMS is one of the major vibroacoustic signal factors;

(vii) Wavelet analysis can be used as a method for the simulation of FSW parameters.

\section{REFERENCES}

[1]G. M. Xie, Z. Y. Ma, and L. Geng, "Development of a fine- grained microstructure and the properties of a nugget zone in friction stir welded pure copper," ScriptaMaterialia, vol. 57, no. 2, pp. 73-76,2007.

[2][2] G. Buffa, L. Fratini, and F. Micari, "A neural network based approach for the design of FSW processes," Key Engineering Materials, vol. 410-411, pp. 413-420,2009.

[3][3] H. Okuyucu, A. Kurt, and E. Arcaklioglu, "Artificial neural network application to the friction stir welding of aluminum plates," Materials and Design, vol. 28, no. 1, pp. 78-84, 2007. 
[4][4] T. Jene, G. Dobmann, G. Wagner, and D. Eifler, "Monitoring of the friction stir welding process to describe parameter effects on joint quality," Welding in the World, vol. 52, no. 9-10, pp. 47-53,2008.

[5][5] E. Castillo, D. P. Morales, A. Garc' ia, F. Mart' inez-Mart' 1, L. Parrilla, and A. J. Palma, "Noise suppression in ECG signals through efficient one-step wavelet processing techniques," Jour- nal of Applied Mathematics, vol. 2013, Article ID 763903, 13 pages, 2013. 